\title{
Kimura Disease With Allergic Bronchopulmonary Aspergillosis: A Case Report
}

\section{Ruiyun Fan}

Nanjing Medical University https://orcid.org/0000-0002-0034-3164

\section{Guopeng Xu}

Nanjing Medical University

\section{Ying Chen}

Nanjing Medical University

Jinghuan Lv

Nanjing Medical University

Zhongwei Zhang ( $\square$ zzhongwei@189.cn )

Nanjing Medical University https://orcid.org/0000-0003-4271-0046

\section{Case report}

Keywords: Kimura disease, Inguinal lymph nodes, Eosinophilia, Immunoglobulin E, Allergic Bronchopulmonary Aspergillosis.

Posted Date: October 18th, 2021

DOl: https://doi.org/10.21203/rs.3.rs-868474/v1

License: (c) (i) This work is licensed under a Creative Commons Attribution 4.0 International License. Read Full License 


\section{Abstract}

Background: Kimura disease (KD) is a rare chronic idiopathic condition of unknown etiology that is prevalent in Asian males. It often causes subcutaneous lumps and enlarged lymph nodes, especially in head and neck region. But KD is also a systemic disease that can involve multiple organs, such as the kidneys and skin.

Case presentation: Here, we report a 62-year-old Chinese man who was characterized by paroxysmal cough, enlarged inguinal lymph nodes, recurrent skin itching, and elevated IgE antibodies specific to $A$. fumigatus. After a comprehensive inspection, the final diagnosis for this patient was KD with Atopic Bronchopulmonary Aspergillosis (ABPA).

Conclusions: The age of onset and the location of the lump involved were not the most common. This study described the patient's diagnosis and treatment process. The coexistence of the two special diseases will provide clinicians with some enlightenment on the etiology of KD. Moreover, we hope to arouse the attention of multidisciplinary team to explore the potential relationship between KD and ABPA. It will contribute to preventing the misdiagnosis and missed diagnosis of KD.

\section{Background}

Kimura's disease is a rare idiopathic, chronic, inflammatory but benign disorder which usually presents as painless subcutaneous masses in the head-neck region. Sometimes it also involves extracutaneous sites, such as salivary glands, lymph nodes, skin, and kidneys. It is endemic to Asia and tend to affect young men aged 20-40 years, with some sporadic cases in non-Asian[1]. Two characteristic laboratory examinations are peripheral eosinophilia and elevated serum immunoglobulin $\mathrm{E}(\lg \mathrm{E})[2]$. The etiology and pathogenesis of KD are still unclear so far. The diagnosis of KD mainly depends on pathological examination. Treatment should aim to relieve symptoms and preserve aesthetics while preventing the recurrences and long-term sequels[3, 4]. In this study, we report a case of KD with ABPA in a Chinese man with special clinical manifestations.

\section{Case Presentation}

A 62-year-old Chinese man, with a history of cough and wheeze after exposed to irritating gases and dust for 50 years, had not received a formal diagnosis and treatment. Four years ago, he presented to the Department of Dermatology because of recurrent itchy skin rashes on the trunk and limbs without obvious inducement. Laboratory tests showed that blood eosinophil count and serum IgE were elevated, then the "eosinophilic dermatoses" was diagnosed. Dermatologists recommended long-term oral prednisolone and cetirizine treatment options. After that, skin pruritus and rashes gradually subsided but still occurred repeatedly. Eight months ago, the patient developed a severe cough and wheezing, and found the right inguinal lymph nodes were swollen without redness, pain, and tenderness. He went to the respiratory department of our hospital. Physical examination showed that the breathing sounds of both 
lungs were thick and a little wheezing could be heard; scattered rashes and markedly pigmentation could be seen on the skin of the whole body, mainly on both lower limbs (Fig. 1); several hard, painless masses about the size of quail eggs could be touched in the right groin area. The patient denied a history of smoking and any family history of malignancy. Hematology revealed total white blood cell(WBC) count of $15.60 \times 10^{\wedge} 9 / /$ (normal: $3.5-9.5 \times 10^{\wedge} 9 / /$ ), a higher eosinophils count of $5.9 \times 10^{\wedge} 9$ (normal: $0.02-$ $0.52 \times 10^{\wedge} 9$ ), and the percentage of eosinophils of $37.8 \%$ (normal: $0.4 \%-8 \%$ ). His tumor markers, antineutrophil cellular antibody (ANCA), anti-nuclear antibody, and blood GM test were negative, but the fungal $\mathrm{G}$ test was positive. Total serum IgE level was $9400 \mathrm{IU} / \mathrm{ml}$ (normal <165) while IgG, IgM, IgA, C3, and $\mathrm{C} 4$ were within the normal range. There was no abnormality in the liver, thyroid, and renal function; coagulation function investigation showed D-dimer was $3.93 \mathrm{mg} / \mathrm{L}$ ) (normal $<0.55 \mathrm{mg} / \mathrm{L}$ ). Pulmonary function examination revealed that FEV1 (Forced expiratory volume in one second) was 1.64L, accounting for $59 \%$ of the predicted value, FEV1/FVC (forced vital capacity) was $85 \%$, and the improvement rate after inhalation of bronchodilator was $15 \%(\geq 12 \%$ indicate a positive bronchodilation test). Chest computed tomography (CT) examination showed increased texture and a little emphysema in both lungs, and multiple pneumatoceles in the right lung. We also found several bronchial walls were thickened and slightly dilated in the lower lobe of lungs (Fig. 2). Vascular ultrasound (US) examination of the vessels showed femoral atherosclerotic plaque formation in both lower extremities. Inguinal ultrasound examination revealed several enlarged lymph nodes in the right groin. We considered that right inguinal lymphoma couldn't be ruled out, so the patient was recommended to undergo a lymph node biopsy. Pathological results showed that complete lymph node structure, lymph node follicular hyperplasia, germinal center enlargement, a large number of eosinophil infiltration and eosinophil abscess in the follicular and interfollicular area, which was consistent with the diagnosis of KD (Fig. 3). Immunohistochemical staining showed that LCA, Vimentin was positive, CD2, CD79a, CD3, CD5, CD 43, CD21, CD23, CD68, Ki-67 was partially positive, S-100 was sporadically positive, and CD1, CD123, Lingren was negative.

Because of his positive fungus $G$ test result and symptoms of bronchial asthma, we suspected that ABPA would be diagnosed. Further inspection revealed that IgE specific to $A$. fumigatus was 1.36KUA/L (normal $<0.1 \mathrm{KUA} / \mathrm{L}$ ). It should be noted that the patient had asthma symptoms and imaging sign of bronchiectasis. Moreover, he met the two necessary conditions of serum total $\mathrm{lgE}>1000 \mathrm{lU} / \mathrm{ml}$ and $A$. fumigatus $\lg \mathrm{E}>0.35 \mathrm{KUA} / \mathrm{L}$, so he was diagnosed as Atopic Bronchopulmonary Aspergillosis[5].

Considering that the enlarged inguinal lymph nodes were small and had no obvious symptoms of discomfort, the patient refused surgical resection and finally accepted conservative treatment. According to his condition, we recommended omalizumab as the first choice. He refused and chose oral prednisone (40 mg/day) combined with itraconazole (200 mg twice daily). The patient was discharged after three weeks, when the dose of prednisone had been reduced to $20 \mathrm{mg}$ per day. Before leaving the hospital, the hematology was checked again. The WBC count was $18.60 \times 10^{\wedge} 9 / /$ with 0.7 percent eosinophils (absolute value: $\left.0.13 \times 10^{\wedge} 9 / \mathrm{l}\right)$. The total serum lgE level was increased $(9400 \mathrm{IU} / \mathrm{ml})$. He had no obvious cough and asthma. The patient was followed up regularly to adjust the dose of the drugs. From January 2021, he started taking "prednisone (10 mg/day) combined with itraconazole (100 mg /day)" for 
maintenance treatment. We plotted the changes of his peripheral blood eosinophil count and serum total IgE into a curve (Fig. 4). The fluctuations were not directly related to the adjustment of the drug dose. His previous enlarged inguinal lymph nodes were currently out of touch and there was no sign of progression. The patient was satisfied with the current curative effect and had a high compliance. No adverse drug reactions and relapses have occurred up to now.

\section{Discussion And Conclusions}

KD was first described as "eosinophilic hyperplastic lymphogranuloma" by Kimm and Szeto (China) in 1937, but it was first reported definitively by Kimura (Japan) in 1948 and has been widely known as Kimura disease since then[6]. It is endemic to Asia and tends to affect young men aged 20-40 years. But it has no strict restrictions on ethnicity, age, and gender. The previous reports have showed that sporadic cases were also found in Spain, Arabia, South America, and other non-Asians. The youngest patient was a 15-month old African American boy[7]. Although it is recognized that two typical laboratory examinations are elevated peripheral eosinophil and serum IgE, Vivek et al. reported a case of KD without peripheral eosinophilia[4]. KD is characterized by subcutaneous masses in the head and neck region, sometimes with local lymph node enlargement and salivary gland involvement. Rare cases can involve the orbit and ocular appendages, epiglottis, armpit, long bone, breast, groin, genitals, mediastinum. In this case, the patient's age was not consistent with the classic age of onset reported in previous literature. Our patient presented rare multiple enlarged inguinal lymph nodes. KD is a systemic disease that can usually involve kidneys and skin, which are usually manifested as nephrotic syndrome, skin pruritus, or rashes, respectively. Our patient had normal liver and kidney function, but there were obvious itching and rashes on the trunk and extremities. It had been reported that eosinophils contain various amounts of tissue factor (TF) and some researchers had concluded that relatively high TF in patients with hypereosinophilia might lead to an increased thrombotic risk[8]. A patient with KD was reported to be complicated with acute limb ischemia and coronary artery disease[9]. The D-dimer in the patient we reported was at a high level. Vascular US examination of both lower extremities showed bilateral femoral atherosclerotic plaques formation, suggesting he had a high risk of thrombosis. Therefore, with the consent of the patient, we prescribed a $0.4 \mathrm{ml}$ heparin sodium injection for prophylactic anticoagulation. The coagulation function result revealed D-dimer was $1.5 \mathrm{mg} / \mathrm{L}$ before he was discharged from the hospital.

$\mathrm{KD}$ is a benign disease of unknown etiology and pathogenesis. However, the increased peripheral eosinophil and serum IgE have oriented that KD might be associated with autoimmunity, insect bites or infections, and allergies caused by parasites[10,11]. To our knowledge, this is the first case of KD combined with ABPA, which led to the hypothesis that Aspergillus fumigatus infection might be one of the causes of KD. But the speculation needs more clinical evidence to confirm. When reviewing the patient's history, we unexpectedly found the patient's chest CT examination showed several enlarged lymph nodes in the left axilla as early December 2018 (Fig. 5a). He didn't have any relative discomfort at that time, it subsided without symptomatic treatment in August 2020 (Fig. 5b). This time the right inguinal lymph nodes enlargement occurred, which manifested KD was self-limited and recurrent with an indolent course and good prognosis[12]. 
The definite diagnosis of KD depends on histopathologic examination. Our initial diagnosis suspicion was lymphoma, but the histopathologic results helped us get an accurate diagnosis. The imaging findings, including $\mathrm{CT}$, magnetic resonance imaging (MRI), and US are non-specific, but they are helpful to determine the lesion morphology, anatomical distribution. Yang et al. indicated that the ill-defined, infiltrative lesions on imaging were associated with a higher recurrence rate[13]. Differential diagnosis of KD includes angiolymphoid hyperplasia with eosinophilia (ALHE), cutaneous IgG4-related disease (IgG4RD), Hodgkin's lymphoma, Langerhans cell histiocytosis, florid follicular hyperplasia, Castleman's disease, dermatopathic lymphadenopathy[14]. The main misdiagnosis is ALHE which shares similar histological features with KD, however, patients with ALHE typically have normal eosinophils and IgE level[15].

There is no standard treatment protocol for KD to date. Treatment include surgical resection, regional or systemic steroids, antihistamines, immunosuppressant including cyclosporine (CsA) or anti-lgE antibody (omalizumab), or radiotherapy[16, 17]. The individualized treatment plan should be selected according to the clinical manifestation of patients. For patients with KD combined with renal involvement, steroids may be the best treatment[18]. Surgical resection is preferred for primary lesions without multiple system involvement[19].

Kimura disease is a chronic disease with a prolonged course and a high recurrence rate. No malignant transformation has been reported till date. KD involves multiple disciplines such as immunology, dermatology, nephrology, and otolaryngology. Its different clinical manifestations have varying degrees of impact on patient's lives, including pruritus, renal function damage, disfigurement, vascular embolism. Rare cases and atypical manifestations of KD make the diagnostic difficulty or be misdiagnosis. Our special case may give some clinicians the inspiration to explore the relationship between KD and ABPA. Multidisciplinary team should pay more attention to collect clinical data to study the etiology of KD.

\section{Abbreviations}

KD: Kimura disease;

ABPA: Atopic Bronchopulmonary Aspergillosis;

IgE: immunoglobulin E;

WBC: white blood cell;

ANCN: anti-neutrophil cellular antibody;

FEV1: Forced expiratory volume in one second;

FVC: forced vital capacity;

CT: computed tomography;

US: ultrasound; 
MRI: magnetic resonance imaging;

TF: tissue factor;

ALHE: angiolymphoid hyperplasia with eosinophilia;

IgG4-RD: IgG4-related disease;

CsA: cyclosporine

\section{Declarations}

\section{Acknowledgements}

The authors thank the patient and his family for their support.

\section{Authors' contributions}

RYF: Collected the imaging date; organized the materials; drafted the manuscript. GPX: Diagnosed; collected the case; provided the methodology; revised the manuscript. YC: Followed up; collected the inspection date. JHL: Provided the pathological technical support and pathological data. ZWZ: Revised the draft; supervised the work. All authors read and approved the final manuscript.

\section{Funding}

No funding.

\section{Availability of data and materials}

Data sharing is not applicable to this article, as no datasets were generated or analyzed during this case report.

\section{Ethics approval and consent to participate}

Not applicable.

\section{Consent for publication}

Informed consent was obtained in writing from patient to publish personal data.

\section{Competing interests}

The authors declare that they have no competing interests.

\section{Author details}


1,2,4 Department of Pulmonary and Critical care, The Affiliated Suzhou Hospital of Nanjing Medical

University, Suzhou Municipal Hospital, Gusu School, Nanjing Medical University, Suzhou 215002, Jiangsu Province, China. ${ }^{3}$ Department of Pathology, The Affiliated Suzhou Hospital of Nanjing Medical University, Suzhou Municipal Hospital, Gusu School, Nanjing Medical University, Suzhou 215002, Jiangsu Province, China.

\section{References}

1. Eh Dam VSK, Mohamad S, Mohamad I: Kimura Disease with Parotid Swelling and Cervical Lymphadenopathy: A Case Report and Literature Review. Medeni Med J 2020, 35(2):170-174.

2. Kuo TT, Shih LY, Chan HL: Kimura's disease. Involvement of regional lymph nodes and distinction from angiolymphoid hyperplasia with eosinophilia. Am J Surg Patho/ 1988, 12(11):843-854.

3. Kumar V, Mittal N, Huang Y, Balderracchi J, Zheng HX, Li Z, Xu Y: A case series of Kimura's disease: a diagnostic challenge. Ther Adv Hematol 2018, 9(7):207-211.

4. Dokania V, Patil D, Agarwal K, Thakur P, Prajapati P: Kimura's Disease without Peripheral Eosinophilia: An Unusual and Challenging Case Simulating Venous Malformation on Imaging Studies-Case Report and Review of literature. J Clin Diagn Res 2017, 11(6):ME01-ME04.

5. Agarwal R, Chakrabarti A, Shah A, Gupta D, Meis JF, Guleria R, Moss R, Denning DW, group Acalw: Allergic bronchopulmonary aspergillosis: review of literature and proposal of new diagnostic and classification criteria. Clin Exp Allergy 2013, 43(8):850-873.

6. Dhingra H, Nagpal R, Baliyan A, Alva SR: Kimura disease: case report and brief review of literature. Med Pharm Rep 2019, 92(2):195-199.

7. AlGhamdi FE, Al-Khatib TA, Marzouki HZ, AlGarni MA: Kimura disease: No age or ethnicity limit. Saudi Med J 2016, 37(3):315-319.

8. Cugno M, Marzano AV, Lorini M, Carbonelli V, Tedeschi A: Enhanced tissue factor expression by blood eosinophils from patients with hypereosinophilia: a possible link with thrombosis. PLoS One 2014, 9(11):e111862.

9. Heo W, Jun HJ, Kang DK, Min HK, Hwang YH, Kim JY, Nam KH: Acute Limb Ischemia and Coronary Artery Disease in a Case of Kimura's Disease. Korean J Thorac Cardiovasc Surg 2017, 50(2):114-118.

10. Sun QF, Xu DZ, Pan SH, Ding JG, Xue ZQ, Miao CS, Cao GJ, Jin DJ: Kimura disease: review of the literature. Intern Med J 2008, 38(8):668-672.

11. Kottler D, Barete S, Quereux G, Ingen-Housz-Oro S, Fraitag S, Ortonne N, Deschamps L, Rybojad M, Flageul B, Crickx B et al: Retrospective Multicentric Study of 25 Kimura Disease Patients: Emphasis on 
Therapeutics and Shared Features with Cutaneous IgG4-Related Disease. Dermatology 2015, 231(4):367377.

12. Li X, Wang J, Li H, Zhang M: Misdiagnosed recurrent multiple Kimura's disease: A case report and review of the literature. Mol Clin Oncol 2019, 10(3):352-356.

13. Lin YY, Jung SM, Ko SF, Toh CH, Wong AM, Chen YR, Chan SC, Cheung YC, Ng SH: Kimura's disease: clinical and imaging parameters for the prediction of disease recurrence. Clin Imaging 2012, 36(4):272278.

14. Zhang X, Jiao Y: The clinicopathological characteristics of Kimura disease in Chinese patients. Clin Rheumatol 2019, 38(12):3661-3667.

15. Abuel-Haija M, Hurford MT: Kimura disease. Arch Pathol Lab Med 2007, 131(4):650-651.

16. Ben-Chetrit E, Amir G, Shalit M: Cetirizine: An effective agent in Kimura's disease. Arthritis Rheum 2005, 53(1):117-118.

17. Miki H, Tsuboi H, Kaneko S, Takahashi H, Yokosawa M, Asashima H, Hirota T, Hagiwara S, Umeda N, Kondo $Y$ et al: A case of refractory Kimura disease with a buccal bulky mass successfully treated with low-dose cyclosporine A: report and review of the literature. Allergol Int 2016, 65(2):212-214.

18. Ren S, Li XY, Wang F, Zhang P, Zhang Y, Li GS, Wang L, Zhong X: Nephrotic syndrome associated with Kimura's disease: a case report and literature review. BMC Nephrol 2018, 19(1):316.

19. Ye P, Ma DQ, Yu GY, Gao Y, Peng X: Comparison of the efficacy of different treatment modalities for Kimura's disease. Int J Oral Maxillofac Surg 2017, 46(3):350-354.

\section{Figures}




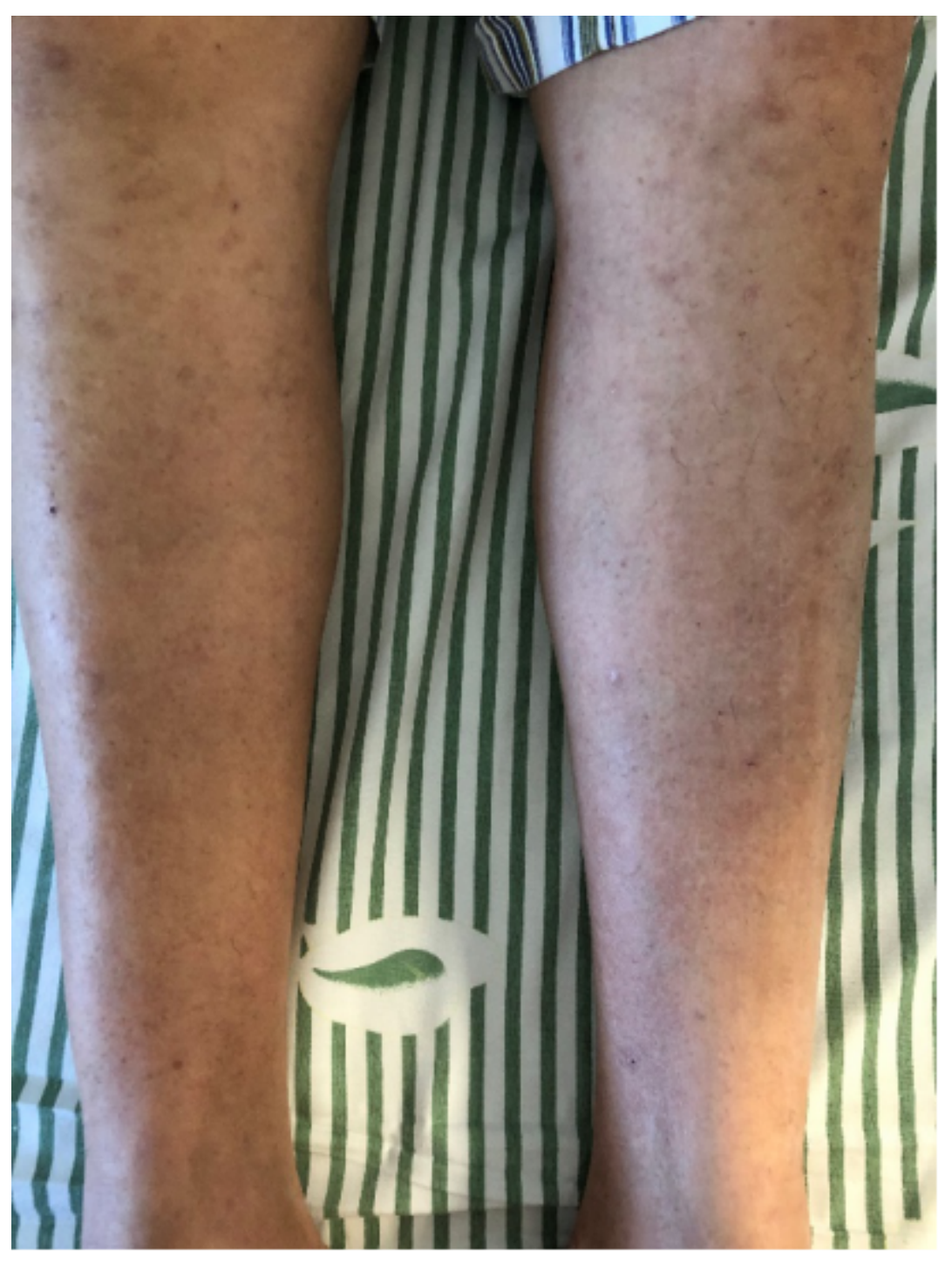

Figure 1

Pigmentation left after red rashes fading on both lower limbs. 

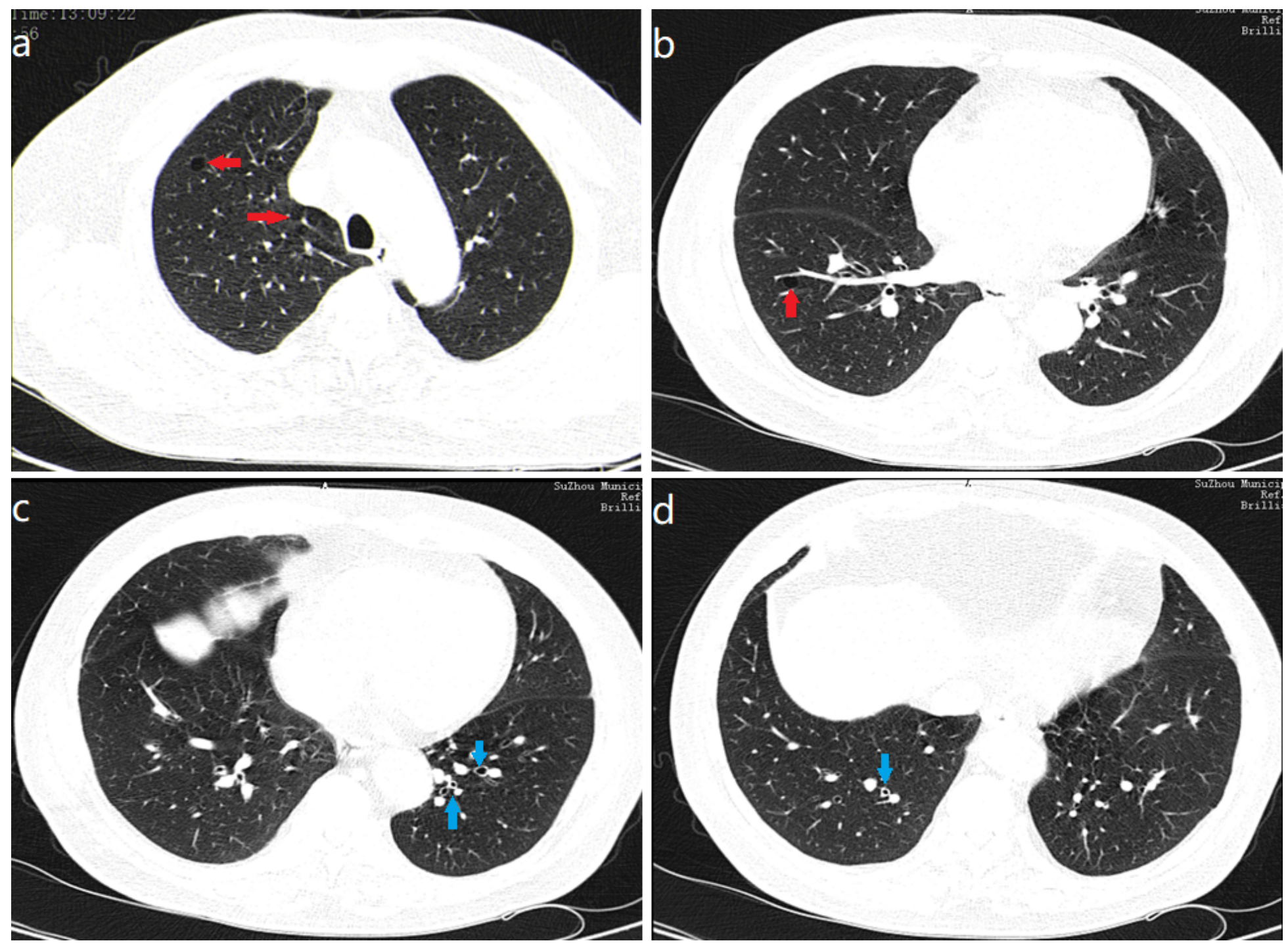

Figure 2

Chest computed tomography (CT) examination: a b Increased texture and a little emphysema in both lungs; multiple pneumatoceles (red arrows). $\mathrm{c} d$ Thickening bronchial walls and mild bronchiectasis in the lower lobe of lungs (blue arrows). 


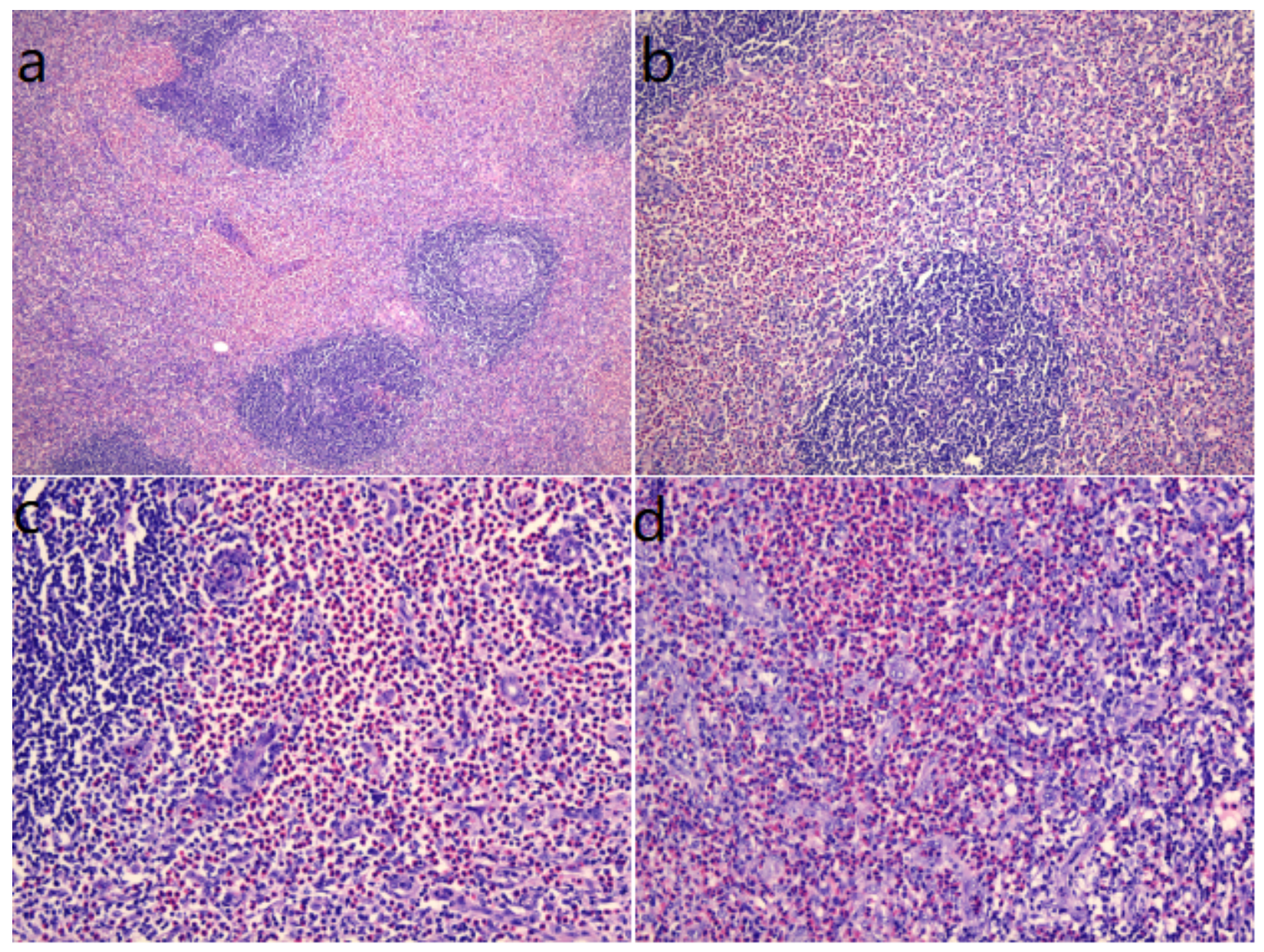

\section{Figure 3}

Hematoxylin-and-eosin-stained sections of the right inguinal lymph node biopsy: a b Complete lymph node structure, lymph node follicular hyperplasia, and germinal center enlargement. c d A large number of eosinophil infiltration and eosinophil abscess in the follicular and interfollicular area.

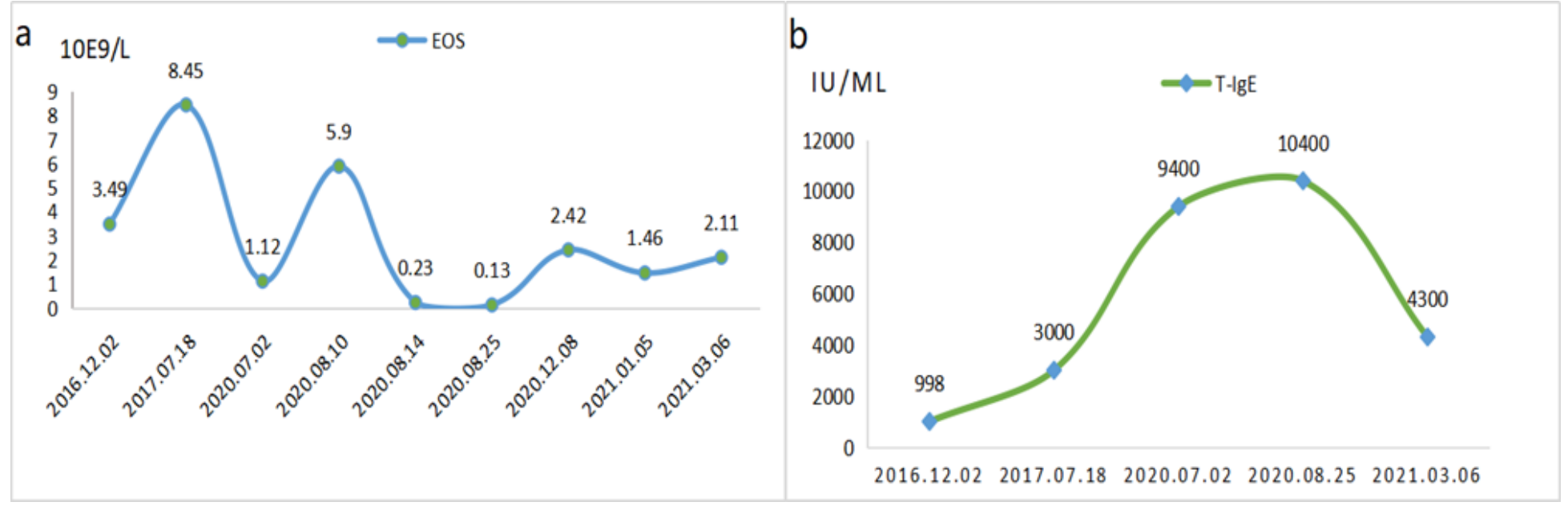

Figure 4

a The change curve of peripheral eosinophils. b The change curve of and serum total IgE Level. 

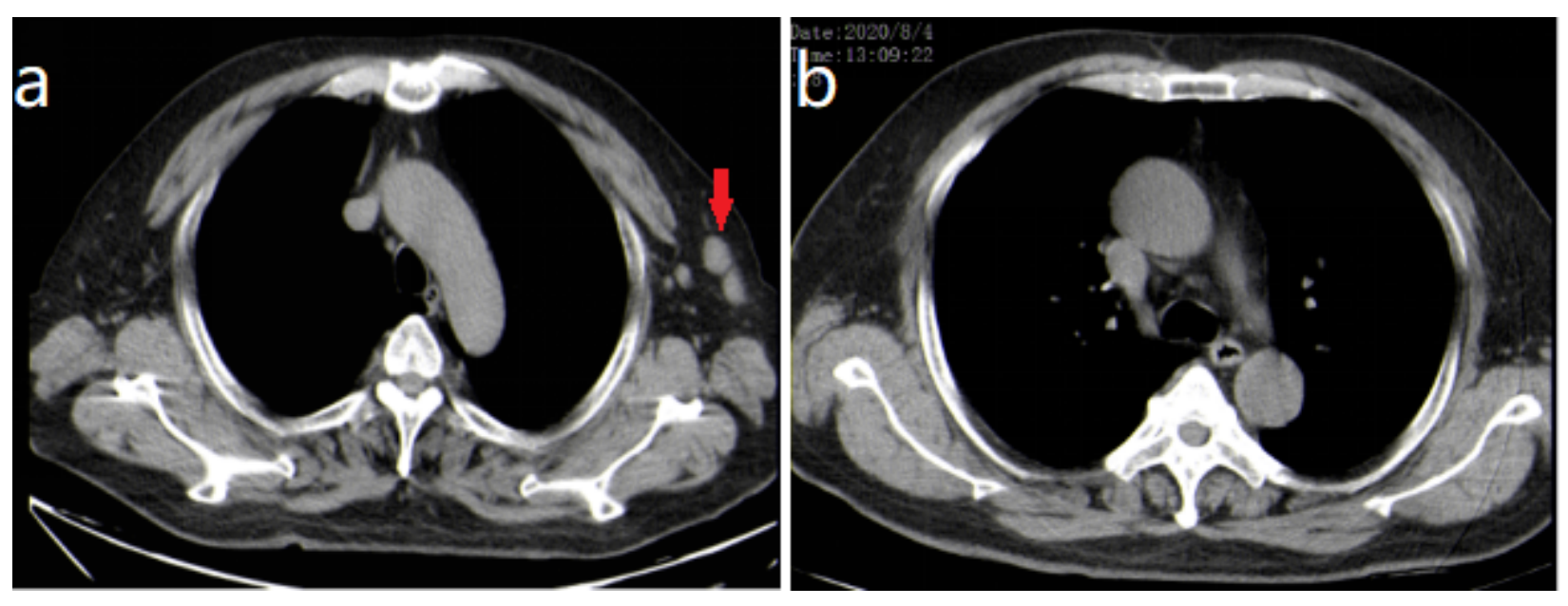

Figure 5

Chest CT scan: a Several enlarged lymph nodes could be seen in the left armpit in December 2018 (red arrow). b No enlarged lymph nodes found in the left armpit in August 2020. 